\title{
Expression of Placental Leucine Aminopeptidase and Adipocyte-Derived Leucine Aminopeptidase in Human Normal and Malignant Invasive Trophoblastic Cells
}

\author{
Kazuhiko Ino, Fumitaka Kikkawa, Takahiro Suzuki, Hiroaki Kajiyama, \\ Kiyosumi Shibata, Seiji Nomura, Atsuo Itakura, Mitsuaki Ito, Tetsuro Nagasaka, \\ Akira Hattori, Masafumi Tsujimoto, and Shigehiko Mizutani
}

Department of Obstetrics and Gynecology (KI, FK, TS, HK, KS, SN, Al, MI, SM), Nagoya University Graduate School of Medicine, Division of Pathology/Clinical Laboratory (TN), Nagoya University Hospital, Showa-ku, Nagoya, and Laboratory of Cellular Biochemistry (AH, MT), RIKEN, Wako, Saitama, Japan

\begin{abstract}
SUMMARY: We recently identified two novel aminopeptidases, placental leucine aminopeptidase (P-LAP) and adipocytederived leucine aminopeptidase (A-LAP). Enzymatically, P-LAP degrades oxytocin, vasopressin, and angiotensin III, while A-LAP degrades angiotensin II and kallidin. In this study we investigated the expression and localization of P-LAP and A-LAP in human trophoblastic cells in the normal placenta $(n=26)$, gestational choriocarcinoma $(n=8)$, and placental site trophoblastic tumor $(n=3)$. On immunoblot analysis both P-LAP and A-LAP proteins were detected in normal placenta and five choriocarcinoma tissues, as well as in two choriocarcinoma cell lines. Immunohistochemical staining of normal placental tissues demonstrated that P-LAP was not only localized in villous syncytiotrophoblasts but also highly expressed in extravillous trophoblasts (EVTs) invading the decidua or maternal spiral arteries. The expression level of P-LAP on these invasive EVTs reached a maximum during the late first to second trimesters of pregnancy, and it decreased in the third trimester. Similarly, A-LAP was strongly expressed in EVTs invading the decidua or spiral arteries in the second trimester of pregnancy, while it was weakly or moderately expressed in villous cytotrophoblasts or EVTs located in the cell columns. These two aminopeptidases were more strongly expressed in all eight choriocarcinomas and three placental site trophoblastic tumors and mainly localized to the intermediate-type trophoblastic tumor cells invading the uterine myometrium or stromal vessels. In summary P-LAP and A-LAP were predominantly expressed in the invasive phenotype of EVTs during placentation, as well as in the invasive tumor cells of trophoblastic neoplasms. These results suggest the involvement of these aminopeptidases in invasiveness of both normal and malignant intermediate-type trophoblasts possibly through degradation of specific peptide substrates. (Lab Invest 2003, 83:1799-1809).
\end{abstract}

$D$ uring early placental development, a dual pathway of trophoblastic differentiation exists. Villous cytotrophoblasts differentiate and fuse to form multinucleated syncytiotrophoblasts, which produce pregnancy-associated hormones and proteins. In contrast, cytotrophoblasts in anchoring villi proliferate to form cell columns and differentiate into intermediate-type trophoblasts, which are called extravillous trophoblasts (EVTs) (Kaufmann and Castellucci, 1997). These EVTs are reported to acquire invasive ability at the distal end of the cell column and infiltrate the decidual tissue into the shallow portion of uterine myometrium during placentation (Zhou et al, 1997). They also invade the maternal spiral arteries in

\section{DOI: 10.1097/01.LAB.0000101728.93907.75}

Received August 7, 2003.

This work was supported in part by Grant-in-Aid No. 15591742 (to KI) from the Japanese Ministry of Education, Culture, Sports, Science and Technology.

Address reprint requests to: $D r$. Kazuhiko Ino, Department of Obstetrics and Gynecology, Nagoya University Graduate School of Medicine, 65 Tsurumai-cho, Showa-ku, Nagoya 466-8550, Japan. E-mail:kazuino@ med.nagoya-u.ac.jp the decidua, which results in the establishment of the maternal-fetal circulation (Pijnenborg et al, 1983). Insufficient or excessive trophoblast invasion occurs in various pathologic conditions, such as pre-eclampsia or trophoblastic neoplasms including choriocarcinoma and placental site trophoblastic tumor (PSTT). Recently, the classification of EVTs has been established, and they are divided into two groups: proliferative phenotype and invasive phenotype (Kaufmann and Castellucci, 1997). The former includes EVTs located in the anchoring cell columns, while the latter consists of two subgroups: interstitial EVTs migrating in the decidua and vascular EVTs invading maternal spiral arteries and replacing endothelial cells.

Although it has been suggested that various factors such as oxygen environment (Caniggia et al, 2000a; Zhou et al, 1998), extracellular matrix components and matrix metalloproteases (Damsky et al, 1992; Huppertz et al, 1998), and several cell adhesion molecules (Damsky et al, 1994; Floridon et al, 2000; Shih et al, 2002; Shih and Kurman, 1996) might be involved in proliferation, invasion, and differentiation of EVTs during the process of implantation and placentation, their regulatory mechanisms are complex and remain con- 
troversial. Recent studies have shown that the human utero-placental unit produces a wide range of important peptides, including endothelin-1, angiotensin II, oxytocin, and gonadotropin-releasing hormone, which require an intricate balance for regulation of trophoblast functions and successful placentation (Cassoni et al, 2001; Chakraborty et al, 2003; Chou et al, 2002; Page et al, 2002; Xia et al, 2002). Thus, cell-surface peptidases, which metabolize bioactive peptides and modulate their receptor binding and signal transduction, might be involved in the regulation of EVT migration and invasion. However, there has been only one report on the association of cell-surface peptidase with EVTs, which showed that dipeptidyl peptidase IV (DPPIV) was expressed in noninvasive EVTs (Sato et al, 2002).

We have purified several peptidases from human placenta, including placental leucine aminopeptidase (P-LAP, EC 3.4.11.3) (Mizutani et al, 1994; Tsujimoto et al, 1992). Our cloning study found that P-LAP is a 165-180-kd type-II membrane-spanning metalloprotease (Rogi et al, 1996), which is a homolog of rat insulin-regulated aminopeptidase (Keller et al, 1995). In addition, we have recently cloned a novel human aminopeptidase termed adipocyte-derived leucine aminopeptidase (A-LAP) from a human adipose tissue cDNA library (Hattori et al, 1999). A-LAP is a 105110-kd zinc-dependent aminopeptidase with significant homology (43\%) to P-LAP, and it is a homolog of mouse puromycin-insensitive leucyl-specific aminopeptidase (Miyashita et al, 2002) or endoplasmic reticulum aminopeptidase associated with antigen processing (ERAP1) (Saric et al, 2002; Serwold et al, 2002). Enzymatically, P-LAP degrades oxytocin, vasopressin, and angiotensin III (Tsujimoto et al, 1992), while A-LAP degrades angiotensin II and kallidin (Hattori et al, 2000). We showed that both P-LAP and A-LAP were widely distributed to various human tissues, including the placenta (Hattori et al, 1999; Rogi et al, 1996), and P-LAP was localized to syncytiotrophoblasts in placental villi (Nagasaka et al, 1997; Nomura et al, 2002). However, to date, our attention has been focused only on these placental aminopeptidases in villous trophoblasts, although their expression and function in EVTs have not yet been studied.

In the present study, we investigated the immunohistochemical localization of P-LAP and A-LAP in human placental trophoblasts, especially focusing on their expression in EVTs at different gestational ages. In addition, we evaluated the expression of these aminopeptidases in two types of trophoblastic neoplasms, choriocarcinoma and PSTT, both of which included neoplastic intermediate-type trophoblastic cells that are phenotypically similar to the EVTs.

\section{Results}

\section{Immunoblot Analysis for Protein Expression of P-LAP and A-LAP}

To examine the protein expression of P-LAP and A-LAP, immunoblot analysis was performed in tropho- blastic tissues and cell lines (Fig. 1). P-LAP protein was detected as an approximately $180-\mathrm{kDa}$ band in second trimester normal placental tissue, while A-LAP protein was detected as a $110-\mathrm{kDa}$ band in the same placental tissue. Tissue samples of five uterine choriocarcinomas (corresponding to patient numbers 1 to 5 in Table 2) were also examined for P-LAP and A-LAP expression by immunoblot analysis. Both of the proteins were detected at various levels in all choriocarcinoma tissues. In addition, both P-LAP and A-LAP were expressed in two choriocarcinoma cell lines, BeWo and JEG3.

\section{Immunohistochemical Expression of A-LAP and P-LAP in EVTs of Normal Pregnancies}

We investigated the expression and localization of A-LAP and P-LAP in EVTs by immunohistochemistry using formalin-fixed, paraffin-embedded tissue sections. EVTs were clearly detected by immunostaining using anti-CK7 mAb, which distinguished EVTs from decidual cells (Fig. 2, A, D, G, and J).

In the first trimester of pregnancy $(n=8)$, A-LAP immunoreactivity was detected in EVTs located in both proximal and distal cell columns of the anchoring villus and also in some interstitial EVTs located in the decidua (Fig. 2B). In contrast, P-LAP immunoreactivity was almost absent in the proximal cell column; however, it was detected in EVTs migrating from the distal cell column into the decidual layers (Fig. 2C). In the second trimester of pregnancy $(n=9)$, both A-LAP and P-LAP were expressed in interstitial EVTs invading the decidua (Fig. 2, E and F). The marked expression of A-LAP and P-LAP were also detected in vascular EVTs existing around the maternal spiral arteries (perivascular EVTs) or invading the vessel walls and replacing the endothelial cells (endovascular

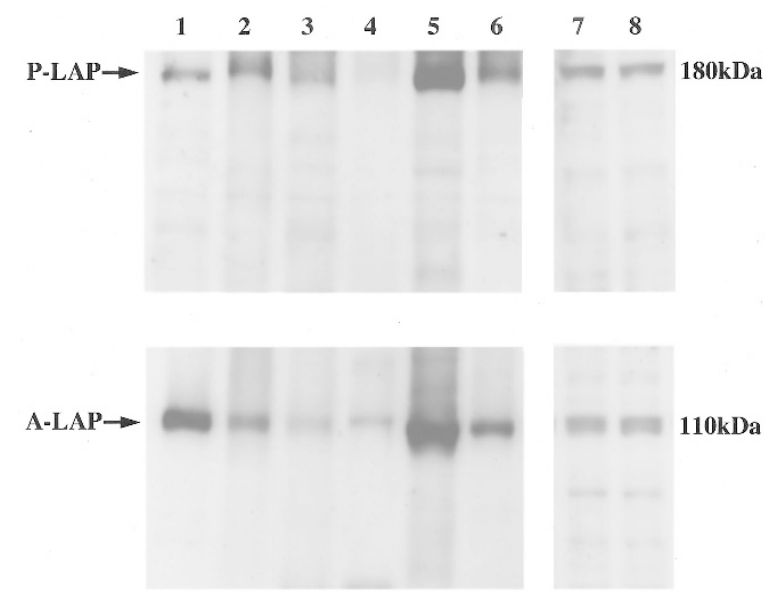

Figure 1.

Immunoblot analysis for protein expression of placental leucine aminopeptidase $(P-L A P)$ and adipocyte-derived leucine aminopeptidase $(A-L A P)$ in trophoblastic tissues and cell lines. P-LAP was detected as a $180-\mathrm{kDa}$ band, while A-LAP was detected as a 110-kDa band. Lane 1, 18-week normal placenta; Lanes 2 to 6 , five different choriocarcinoma tissues obtained from patient numbers 1 to 5 as shown in Table 2; Lane 7, BeWo cells; and Lane 8 JEG3 cells. 
EVTs) (Fig. 2, $\mathrm{H}$ and I). In the third trimester of pregnancy $(n=9)$, both aminopeptidases were still detected in some interstitial and vascular EVTs at weak or focally moderate levels (Fig. 2, K and L). The expression pattern of these aminopeptidases was mainly cytoplasmic but occasionally mixed with the membranous pattern.

The expression and localization of A-LAP and $P$-LAP in villous trophoblasts and EVTs are summarized in Table 1. In floating villi, P-LAP was stably expressed at a moderate level on the apical surface of syncytium throughout gestation as previously reported (Nomura et al, 2002), whereas A-LAP was weakly expressed in cytotrophoblasts. In contrast, both aminopeptidases were strongly expressed in the invasive phenotype of EVTs invading the decidua or maternal spiral arteries during the process of placentation from the first to the second trimester of pregnancy, although A-LAP was also expressed in the proliferative EVTs of the cell columns at weak to moderate levels.

\section{Immunohistochemical Expression of A-LAP and P-LAP in Choriocarcinoma and PSTT}

To examine whether A-LAP and P-LAP were expressed not only in EVTs of normal pregnancy but also in neoplastic trophoblasts of gestational trophoblastic tumors, immunohistochemical analysis was performed on formalin-fixed, paraffin-embedded tissue sections of choriocarcinoma $(n=8)$ and PSTT $(n=3)$.

In noninvasive lesions of uterine choriocarcinoma, immunoreactivities of A-LAP and P-LAP were weakly or moderately detected in the cytoplasm of the intermediate and cytotrophoblastic but not syncytiotrophoblastic, choriocarcinoma cells (Fig. 3, A and B). In the lesions of vascular invasion of intermediate-type choriocarcinoma cells, both A-LAP and P-LAP were strongly expressed (Fig. 3, C and D). Both aminopeptidases were also strongly expressed in the intermediate-type choriocarcinoma cells at the invasive edge of lung metastasis lesions (Fig. 3, E and F).

In the lesions of PSTT, A-LAP and P-LAP were focally expressed in mononuclear or occasionally multinucleated intermediate trophoblastic cells (Fig. 4, A and B). In contrast, both aminopeptidases were strongly and diffusely expressed in PSTT cells infiltrating uterine myometrium in a scattered pattern or invading some stromal vessels (Fig. 4, C and D). Finally, in lung metastasis lesions of PSTT, both enzymes were localized to the invading PSTT cells that surrounded adjacent vessels (Fig. 4, E and F).

The staining pattern in choriocarcinoma and PSTT was predominantly cytoplasmic and granule-like but focally membranous, which was similar to the pattern shown in invasive EVTs. The results of immunohistochemical expression are summarized in Table 2. Both aminopeptidases were expressed in all eight choriocarcinomas and in all three PSTTs examined, including the lung or liver metastasis lesions. In most cases the expression levels were strongly or moderately positive and weakly positive in only one case for each aminopeptidase. There was no specific difference in the expression pattern and intensity between the two aminopeptidases in choriocarcinoma and PSTT.

\section{Subcellular Localization of P-LAP in Trophoblastic Cells}

To clarify the subcellular localization of P-LAP, placental tissue homogenate was fractionated by differential centrifugation, and immunoblot analysis was performed. As shown in Figure 5, the bulk of P-LAP was found in the microsomal fraction, with a minimal amount in the cytosolic fraction. Furthermore, immunofluorescence staining of BeWo cells showed that $\mathrm{P}$-LAP was located mainly in vesicular structures in the cytoplasm, partially similar to the KDEL localization in the endoplasmic reticulum (ER), but the enzyme was also focally detected on the cell membrane (Fig. 6). These results suggest that P-LAP is localized on the membrane of intracellular vesicles and could be translocated to the cell surface, while A-LAP is mainly located in the ER vesicle as an ER aminopeptidase as previously reported (Saric et al, 2002).

\section{Discussion}

In the current study, we demonstrated immunohistochemical expression and localization of the recently cloned two novel aminopeptidases, P-LAP and A-LAP, in trophoblastic cells in normal placenta as well as in trophoblastic tumors. As we previously reported, P-LAP was expressed on the apical surface of villous syncytium at a stable level throughout gestation, where P-LAP is considered to work as an oxytocinase for the regulation of labor pains (Nomura et al, 2002). In addition, our immunohistochemistry demonstrated that A-LAP was weakly or focally expressed on cytotrophoblasts in the floating villi, although its significance remains to be clarified.

In contrast to their differential localization in villous trophoblasts, both P-LAP and A-LAP were predominantly localized to the interstitial EVTs migrating in the decidua and also to the vascular EVTs invading the maternal spiral arteries. Furthermore, the expression levels of P-LAP and A-LAP on the invasive phenotype EVTs increased in the late first trimester of pregnancy and reached a maximum in the second trimester, and then decreased to lower levels in the third trimester. It is well known that the maximal invasive activity of interstitial EVTs occurs in the late first trimester peaking at around 10 to 12 weeks (Caniggia et al, 2000b), and the invasion of vascular EVTs into the spiral arteries is established at around 16 to 18 weeks (Pijnenborg et al, 1983). Therefore, the expression and localization of the two aminopeptidases presented in our study appear to be associated with EVT migration and invasion during the process of placentation.

It remains to be clarified how P-LAP and A-LAP contribute to the regulation of EVT functions. Enzymatically, aminopeptidases commonly hydrolyze $\mathrm{N}$-terminal amino acids of peptide substrates, but each aminopeptidase has a strict substrate specificity. Namely, P-LAP specifically degrades oxytocin, vaso- 


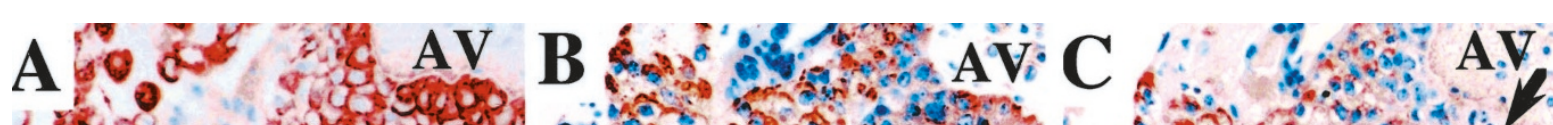

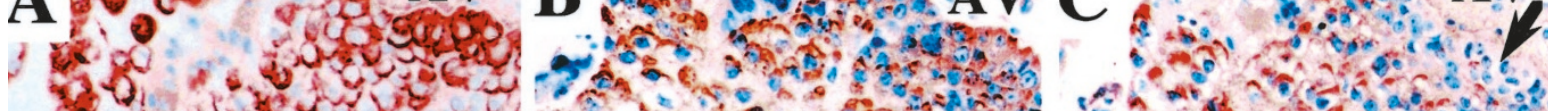

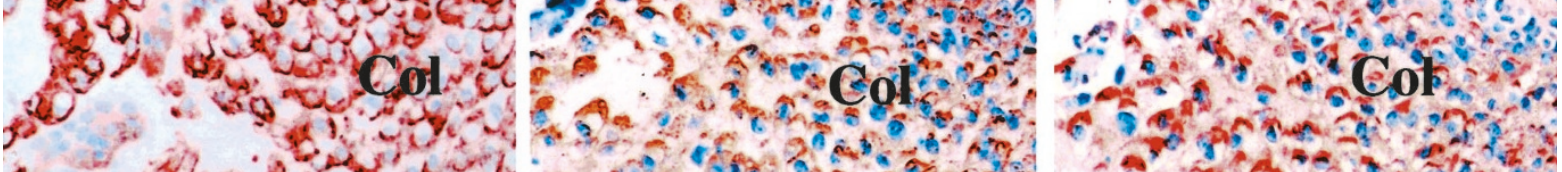

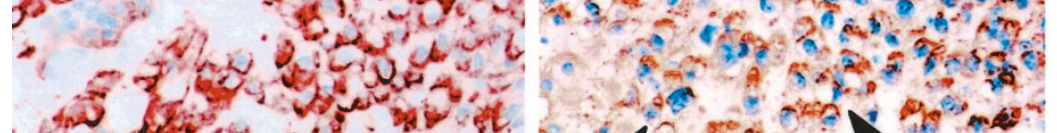

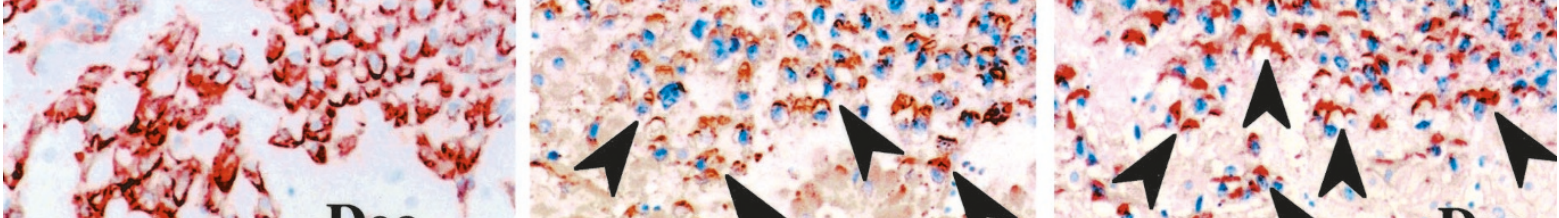

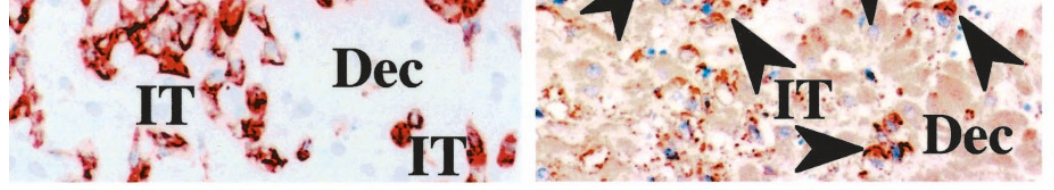
$\operatorname{lin}^{A}$

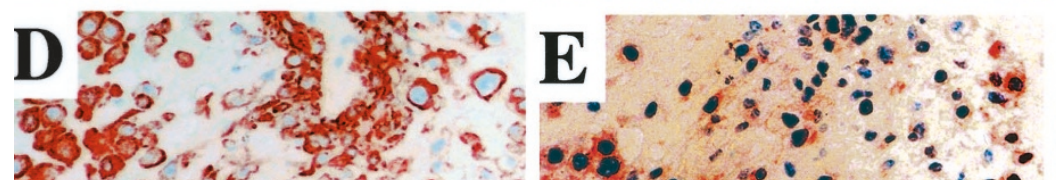
(3) $4, \because \because \%$ 3.

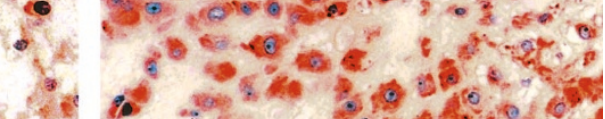
- ro

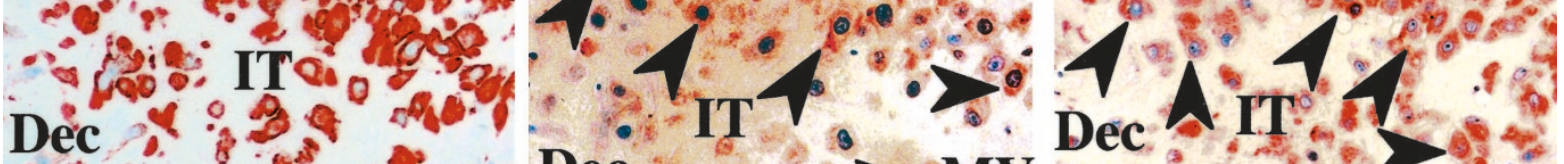
of 50 . Dec, , > > $F$...

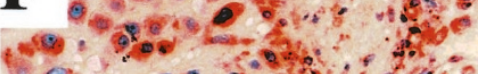

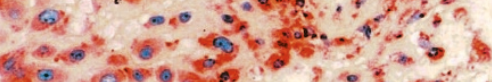
(1) Gons

G

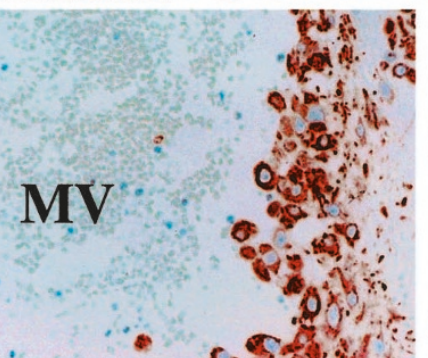

2. fort. 4 .

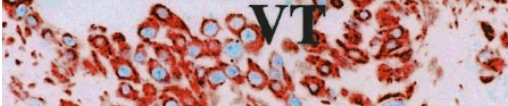

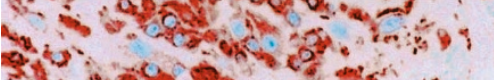

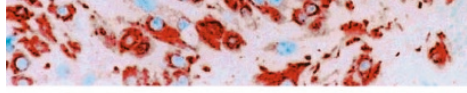

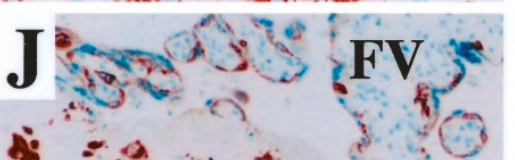

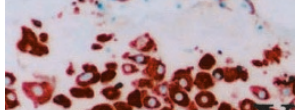

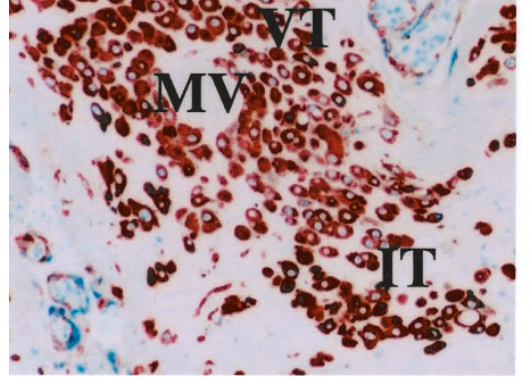

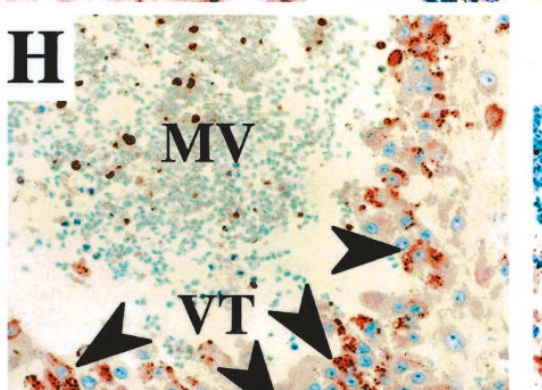

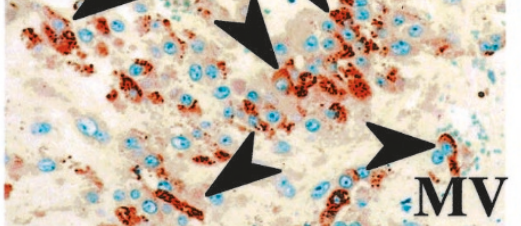

ge.
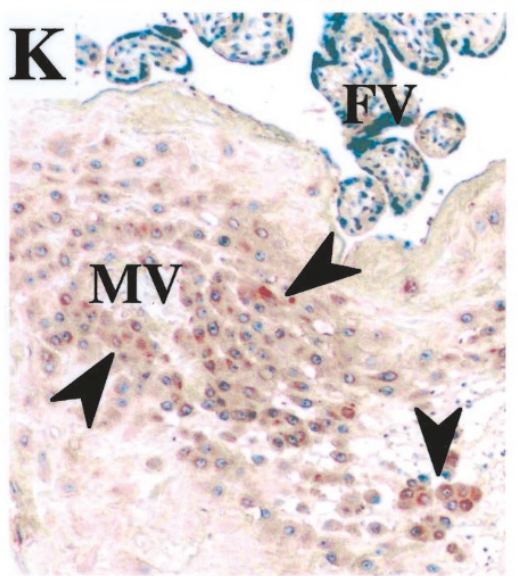

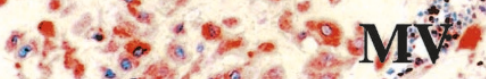

I $3 \mathrm{Na}, 6$,

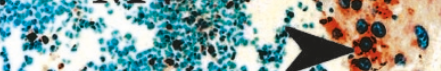
Prot in $1^{2}+0^{\circ}$

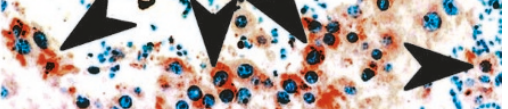
- $0^{\circ}: 0.0$

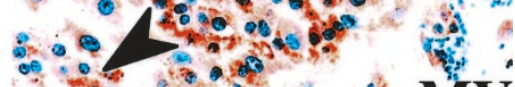
8.0.8 MV
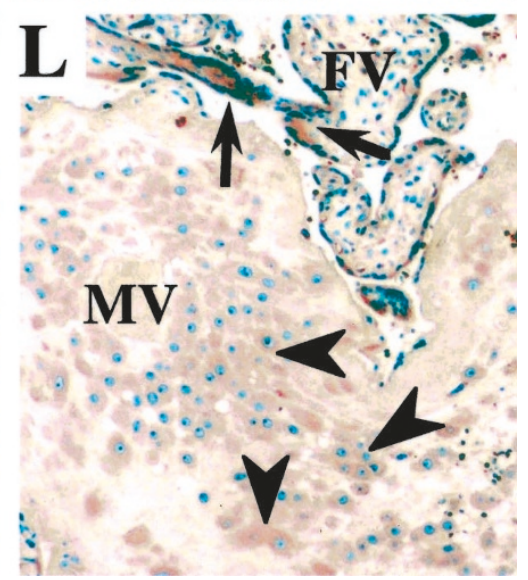
Table 1. Expression and Localization of A-LAP and P-LAP in VT and EVT in the First, Second, and Third Trimester of Pregnancy by Immunohistochemistry ${ }^{a}$

\begin{tabular}{|c|c|c|c|c|c|c|c|}
\hline & \multicolumn{2}{|c|}{ Villous trophoblast } & \multicolumn{2}{|c|}{$\begin{array}{c}\text { Proliferative } \\
\text { phenotype EVT }\end{array}$} & \multicolumn{3}{|c|}{ Invasive phenotype EVT } \\
\hline & Syncytium & Cytotrophoblast & $\begin{array}{l}\text { Proximal } \\
\text { column }\end{array}$ & $\begin{array}{l}\text { Distal } \\
\text { column }\end{array}$ & Interstitial & Perivascular & Endovascular \\
\hline \multicolumn{8}{|c|}{ First trimester $(n=8)^{b}$} \\
\hline A-LAP & - & + & + & + & + & $+1-$ & nd \\
\hline P-LAP & + & - & - & + & ++ & $+/-$ & nd \\
\hline \multicolumn{8}{|c|}{ Second trimester $(n=9)$} \\
\hline A-LAP & - & + & + & + & ++ & ++ & + \\
\hline P-LAP & + & - & - & + & ++ & ++ & ++ \\
\hline \multicolumn{8}{|c|}{ Third trimester $(n=9)$} \\
\hline A-LAP & - & $+1-$ & nd & nd & + & + & + \\
\hline P-LAP & + & - & nd & nd & $+1-$ & + & + \\
\hline
\end{tabular}

A-LAP, adipocyte-derived leucine aminopeptidase; EVT, extravillous trophoblast; P-LAP, placental leucine aminopeptidase; VT, villous trophoblast.

${ }^{a}$ Expression intensity was scored as follows: - , negative (no positive cells); $+/-$, weakly or focally positive $(<30 \%$ positive cells); + , moderately positive $(30 \%$ to $70 \%$ positive cells); ++ , strongly or diffusely positive ( $>70 \%$ positive cells); nd, not determined because of the absence of trophoblasts in this site.

${ }^{b}$ Four samples with gestational ages $<9$ wk obtained from dilation and currettage could not be evaluated on invasive EVTs because of lack of consecutive sections from anchoring villi to decidua.

Table 2. Immunohistochemical Expression of A-LAP and P-LAP in Neoplastic Trophoblasts of Gestational Choriocarcinomas and PSTTs ${ }^{a}$

\begin{tabular}{rcccc}
\hline $\begin{array}{c}\text { Patient } \\
\text { no. }\end{array}$ & $\begin{array}{c}\text { Pathologic } \\
\text { diagnosis }\end{array}$ & Site & A-LAP & P-LAP \\
\hline 1 & Choriocarcinoma & Uterus & + & + \\
2 & Choriocarcinoma & Uterus & + & ++ \\
3 & Choriocarcinoma & Uterus & + & + \\
4 & Choriocarcinoma & Uterus & ++ & ++ \\
5 & Choriocarcinoma & Uterus & + & ++ \\
6 & Choriocarcinoma & Uterus & $+/-$ & + \\
7 & Choriocarcinoma & Lung metastasis & ++ & ++ \\
8 & Choriocarcinoma & Liver metastasis & + & $+/-$ \\
9 & PSTT & Uterus & ++ & ++ \\
10 & PSTT & Uterus & + & + \\
11 & PSTT & Lung metastasis & ++ & ++ \\
\hline
\end{tabular}

A-LAP, adipocyte-derived leucine aminopeptidase; P-LAP, placental leucine aminopeptidase; PSTT, placental site trophoblastic tumor.

${ }^{a}$ Expression intensity was scored as follows: - , negative (no positive cells); $+/-$, weakly or focally positive $(<30 \%$ positive cells); + , moderately positive ( $30 \%$ to $70 \%$ positive cells); ++ , strongly or diffusely positive (> 70\% positive cells).

pressin, and angiotensin III (Tsujimoto et al, 1992), while A-LAP degrades angiotensin II and kallidin (precursor of bradykinin) (Hattori et al, 2000). Among the possible target peptides, angiotensin II and its receptors are present in both EVTs and decidual cells as the local renin-angiotensin system (Cooper et al, 1999; Li et al, 1998; Vinson et al, 1997). Furthermore, Xia et al (2002) reported that angiotensin II inhibited human trophoblast invasion via synthesis of plasminogen activator inhibitor-1. Because both P-LAP and A-LAP are involved in the metabolism of angiotensins, these aminopeptidases could modulate the local concentrations of angiotensins and their receptor bindings, which may contribute to the regulation of EVT migration and invasion. It is also speculated that both aminopeptidases expressed on endovascular EVTs in spiral arteries may contribute to the vasodilation and maintenance of uteroplacental blood flow through inactivation of angiotensin II (vasoconstrictor) and generation of bradykinin (vasodilator). Contrary to P-LAP or A-LAP, DPPIV, the aminopeptidase cleaving certain chemokines, was selectively expressed in the noninvasive phenotype EVTs, and down-regulation of DPPIV was associated with EVT invasion (Sato et al, 2002). Our previous studies also showed that neutral endopeptidase (NEP), another peptidase degrading endothelin-1 that is one of the peptides promoting EVT invasion (Chakraborty et al, 2003), was expressed in differentiated syncytiotrophoblasts but not in invasive EVTs (Ino et al, 2000; Uehara et al, 2001). Thus, whether these peptidases function as a positive or negative regulator of the invasiveness of EVT appears to be dependent on the type of target peptide sub-

Figure 2.

Immunohistochemical expression of adipocyte-derived leucine aminopeptidase (A-LAP) and placental leucine aminopeptidase (P-LAP) in extravillous trophoblasts (EVTs) of normal pregnancies at 12-wk (A to C), 15-wk (D to F), 18-wk ( $\mathrm{G}$ to I), and 37-wk gestation (J to L). EVTs were identified by immunostaining with anti-CK7 antibody (A, D, G, and J). At 12-wk gestation, A-LAP and P-LAP were predominantly expressed in EVTs at the distal cell columns and in some interstitial EVTs (arrowheads in B and C), while P-LAP immunoreactivity in the proximal cell columns was almost absent (arrow in C). At 15-wk gestation, both A-LAP and P-LAP were strongly expressed in interstitial EVTs invading the decidua (arrowheads in E and F). At 18-wk gestation, A-LAP and P-LAP were detected in vascular EVTs around/intra maternal spiral vessels (arrowheads in $\mathrm{H}$ and I). At 37-wk gestation, both aminopeptidases were expressed in some vascular and interstitial EVTs at low levels (arrowheads in $\mathrm{K}$ and L), while P-LAP was detected in syncytiotrophoblasts in floating villi (arrow in L). $A V=$ anchoring villus; $C o l=c e l l ~ c o l u m n ; ~ D e c=$ decidua; $I T=$ interstitial $\mathrm{EVT} ; M V=$ maternal vessel; $V T=$ vascular EVT; $F V=$ floating villi. Original magnifications: $\times 200$ (A to I) and $\times 100(\mathrm{~J}$ to $\mathrm{L}$ ). 

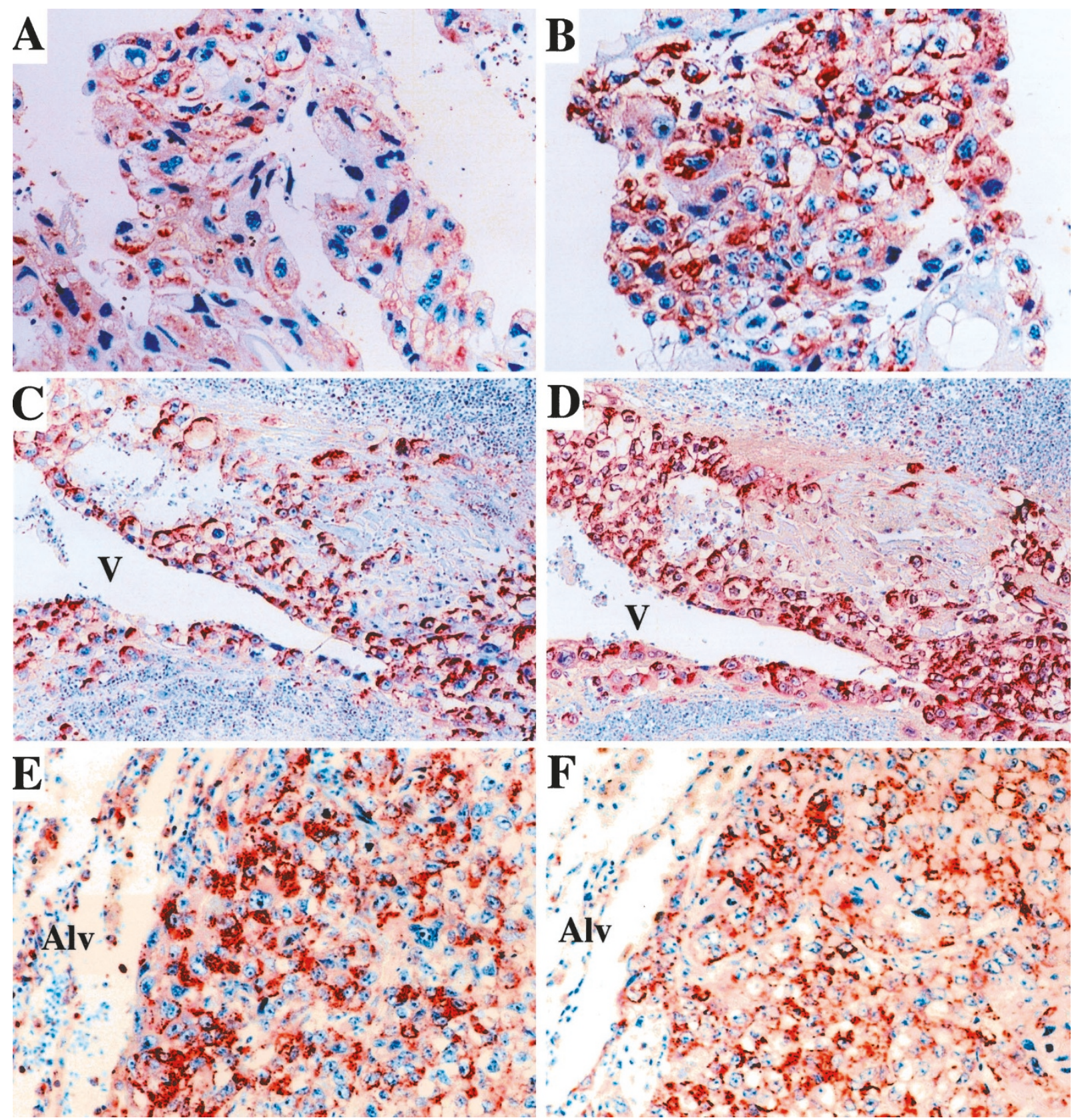

Figure 3.

Immunohistochemical expression of adipocyte-derived leucine aminopeptidase (A, C, and E) and placental leucine aminopeptidase (B, D, and F) in choriocarcinoma tissues. ( $A$ and B) Noninvasive lesions of uterine choriocarcinoma. ( $C$ and D) Choriocarcinoma invading the myometrium and stromal vessels. ( $E$ and $F$ ) Lung metastasis lesions showing invasion into alveolar tissues. Both aminopeptidases were predominantly expressed in invading intermediate-type choriocarcinoma cells. $A / v=$ alveolar tissues; $V=$ vessels. Original magnifications: $\times 200(A, B, E$, and $F)$ and $\times 100(C$ and $D)$

strates. Furthermore, the balance among the peptidases may be important for successful placentation.

Our present data showed that both P-LAP and A-LAP were expressed in all choriocarcinoma and PSTT tissues examined, and their expression levels were mostly higher than those of EVTs. Furthermore, both aminopeptidases were predominantly localized to intermediate trophoblastic cells invading the uterine myometrium, stromal vessels, or lung tissues. These results suggest that P-LAP and A-LAP may be involved not only in the normal EVT invasion but also in the invasion of neoplastic trophoblast. We have re- cently reported that overexpression of P-LAP in endometrial cancer cells showed a higher growth and invasive activity (Suzuki et al, 2003). In addition, PLAP-transfected ovarian cancer cells showed a higher invasive activity via increasing matrix metalloproteases-2 expression (Shibata et al, unpublished data). On the other hand, we reported that overexpression of DPPIV in ovarian cancer cells resulted in decreased invasive potential and reduced matrix metalloproteases-2 expression (Kajiyama et al, 2002, 2003). Our preliminary experiment also showed that DPPIV was weakly or faintly expressed in invasive lesions of 

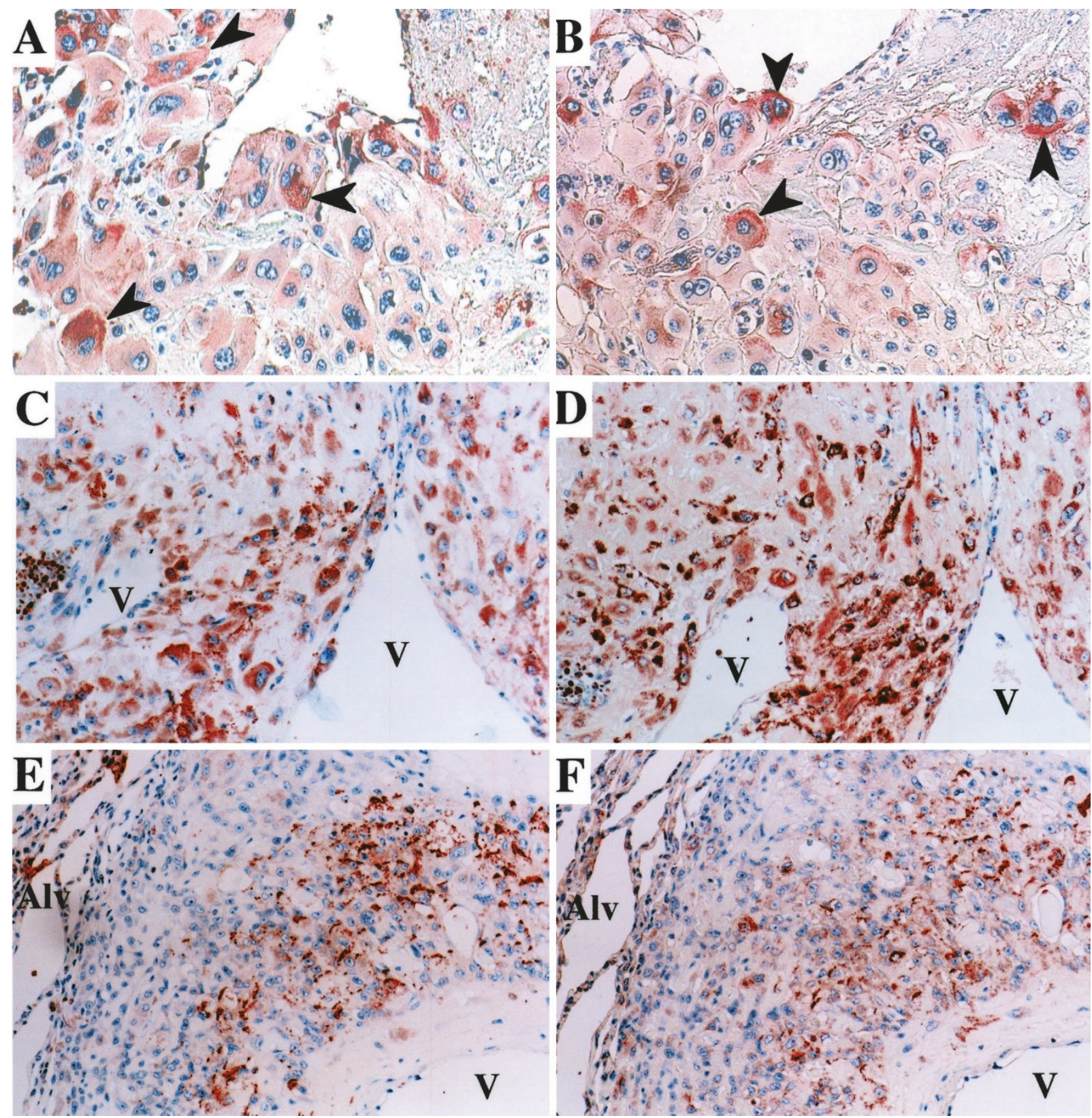

\section{Figure 4.}

Immunohistochemical expression of adipocyte-derived leucine aminopeptidase ( $\mathrm{A}, \mathrm{C}$, and $\mathrm{E})$ and placental leucine aminopeptidase $(\mathrm{B}, \mathrm{D}$, and $\mathrm{F})$ in placental site trophoblastic tumor (PSTT) tissues. (A and B) Uterine lesions of PSTT showing focally positive PSTT cells (arrowheads). (C and D) Lesions of scattered invasion into myometrium and stromal vessels ( $V$. ( $E$ and $F$ ) Lung metastasis lesions showing invasion into alveolar (A/v) tissues and adjacent vessels. Both aminopeptidases were strongly expressed in invading PSTT cells. Original magnifications: $\times 400(A$ and $B), \times 100$ (C to F).

choriocarcinoma (Ino et al, unpublished data). Taken together, it appears that P-LAP has an invasionstimulatory effect, while DPPIV has an adverse effect. Furthermore, recent studies have shown that puromycin-insensitive leucyl-specific aminopeptidase, a mouse homolog of A-LAP, induced cell spreading via activation of integrins (Akada et al, 2002). Needless to say, the invasion of normal EVTs is strictly regulated to prevent extension beyond onethird of the myometrium, while extensive and uncontrolled invasion into the myometrium occurs in both choriocarcinoma and PSTT. The reason for this difference is still unknown, but it might be due, at least in part, to higher expression of P-LAP and A-LAP in neoplastic trophoblasts than in normal EVTs, as well as to an imbalance between the invasion-promoting and -inhibiting peptides or their degrading peptidases in trophoblastic neoplasms.

It is of interest that the staining pattern of P-LAP and A-LAP in trophoblastic cells was predominantly cytoplasmic but focally mixed with the membranous pattern. Furthermore, P-LAP was detected in the microsomal fraction of placental tissue homogenate, and it was mainly localized to some vesicular structures in the cytoplasm and also focally detected on the cell membrane by immunofluorescence staining. P-LAP is 


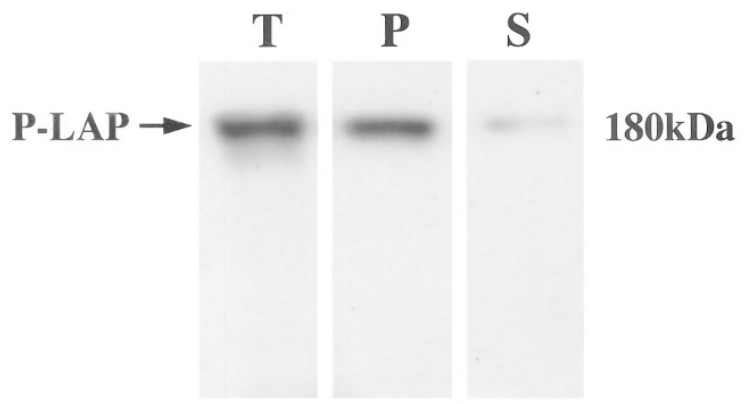

Figure 5.

Subcellular localization of placental leucine aminopeptidase ( $P$-LAP) on immunoblot analysis. Placental tissue homogenate $(T)$ was fractionated by differential centrifugation, and the microsomal (pellet; $P$ ) and cytosolic (supernatant; $S$ ) fractions were isolated as described in "Materials and Methods."

a homolog of rat insulin-regulated aminopeptidase, which is colocalized with glucose transporter 4 in intracellular vesicles and translocated to the cell surface in response to insulin (Keller et al, 1995; Ross et al, 1996). Similarly, we demonstrated that human P-LAP was also translocated to the cell surface in response to oxytocin stimulation (Nakamura et al, 2000). In contrast, A-LAP was proved to be a homolog of ERAP1, which is located in the ER vesicles and involved in peptide trimming for antigen presentation (Saric et al, 2002; Serwold et al, 2002). From these findings, both P-LAP and A-LAP are considered located to the vesicular structures in the cytoplasm of trophoblasts, and may be translocated to the cell surface in response to target peptide stimulation. Further studies are necessary to clarify the detailed subcellular localization of these aminopeptidases and their translocation systems in trophoblastic cells.

In conclusion we demonstrated that P-LAP and A-LAP were highly expressed in the invasive phenotype of EVTs during placentation of normal pregnancy, as well as in the invasive neoplastic intermediate trophoblasts of choriocarcinoma and PSTT. Although trophoblastic invasion is controlled by numerous factors, our findings suggest that it is regulated, at least in part, by aminopeptidases that degrade specific bioactive peptides. Identification of the functional roles of P-LAP and A-LAP in both normal and malignant trophoblastic cells could facilitate further insights into the behavior of trophoblasts in normal implantation/ placentation and in various pathologic conditions such as pre-eclampsia or trophoblastic neoplasms.

\section{Materials and Methods}

\section{Cell Lines}

Two choriocarcinoma cell lines, BeWo and JEG3, were obtained from the American Type Culture Collection (Manassas, Virginia). Cells were maintained in a monolayer culture in RPMI 1640 medium (Sigma Chemical Company, St. Louis, Missouri) supplemented with $10 \%$ heat-inactivated FCS, penicillin (100 $\mathrm{U} / \mathrm{ml})$, and streptomycin $(100 \mu \mathrm{g} / \mathrm{ml})$ at $37^{\circ} \mathrm{C}$ in a humidified $5 \% \mathrm{CO}_{2}$ atmosphere.
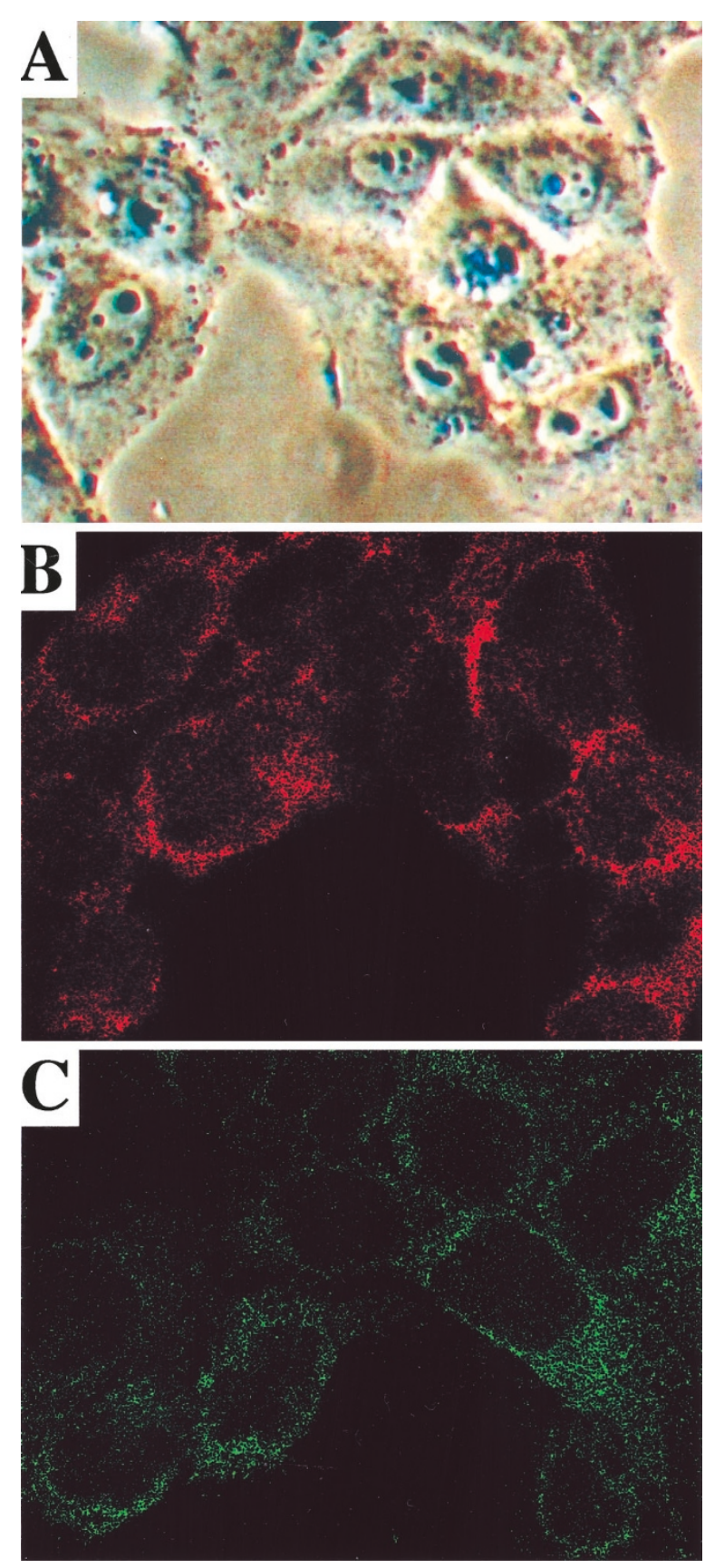

Figure 6.

Immunofluorescence staining of BeWo cells. (A) BeWo cells observed by phase contrast microscopy. Immunofluorescence staining by confocal laser microscopy for placental leucine aminopeptidase (P-LAP) (B) and KDEL, a specific marker of the endoplasmic reticulum (C). P-LAP was mainly located in vesicular structures in the cytoplasm, partially similar to the KDEL, and also focally detected on the cell membrane.

\section{Tissues}

Placental tissues were obtained from normal uncomplicated pregnancies (total $n=26$ ) by legal induced abortions or selective cesarean sections. Gestational ages for each sample were as follows: first trimester ( $n$ $=8$ ): 6 weeks (2 samples), 7 weeks, 8 weeks, 10 weeks, 11 weeks (2), and 12 weeks; second trimester $(n=9)$ : 14 weeks (2), 15 weeks (2), 16 weeks (2), 18 weeks, 20 weeks, and 22 weeks; and third trimester ( $n$ 
$=9$ ): 30 weeks, 32 weeks, 34 weeks, 36 weeks, 37 weeks, 39 weeks (2), and 40 weeks (2). Samples with pathologically abnormal findings, such as apparent viral and bacterial infections, placental infarctions, and any structural anomalies, were excluded from this study. Tissue samples of gestational choriocarcinoma $(n=8)$ and PSTT $(n=3)$ were obtained from patients who underwent surgical treatment at Nagoya University Hospital. Informed consent for the use of tissue was obtained from each patient. The use of samples was also approved by the institutional review board of Nagoya University Hospital. Tissue samples were fixed in $10 \%$ formalin, embedded in paraffin, and routinely stained with hematoxylin and eosin for histologic examination. Some of the samples were snapfrozen in liquid nitrogen for protein extraction.

\section{Antibodies}

Mouse mAb against cytokeratin 7 (CK7, Clone OV-TL $12 / 30$ ), a marker for epithelial cells that is widely used for distinguishing EVTs from decidual cells (Blaschitz et al, 2000), was purchased from DAKO Corporation (Carpinteria, California). The rabbit anti-P-LAP polyclonal antibody was raised against the $\mathrm{N}$-terminal cytoplasmic domain N28 (amino acid sequence 55-82 of human P-LAP) (Nakanishi et al, 2000). Briefly, antiserum against $\mathrm{N} 28$ was raised by immunization of rabbits with the GST-P-LAP fusion protein, and a specific antibody was affinity-purified using an N28 antigen-coupled agarose resin column. Using the purified N28-specific antibody, a single 180-kDa immunoreactive band of P-LAP was detected in placental homogenate on immunoblot analysis, and the band disappeared when antibody was preabsorbed with N28 antigen (Nakanishi et al, 2000). The rabbit anti-ALAP polyclonal antibody was raised against a synthetic peptide corresponding to the C-terminal 15 amino acids (C15) of human A-LAP, and the rabbit immunization and immunoaffinity purification were performed using the same procedure (Hattori et al, 1999).

\section{Immunoblot Analysis}

Cells or tissues were washed with PBS and lysed in a lysis buffer (20 mm Tris-HCl pH 7.5, 2 mm EDTA, 150 $\mathrm{mm} \mathrm{NaCl}, 1 \%$ Triton $\mathrm{X}-100$, and protease inhibitor mixture tablets). After centrifugation for 10 minutes at $15,000 \times g$, the supernatant was assayed for protein concentration using a BCA kit (Pierce, Rockford, Illinois). Ten $\mu \mathrm{g}$ of protein was equally diluted in a sample buffer and denatured at $95^{\circ} \mathrm{C}$ for 5 minutes. Proteins were separated by SDS-PAGE and transferred onto nitrocellulose membranes. Membranes were blocked with $5 \%$ skim milk, and then immunoblotted with anti-P-LAP antibody (1:1000 dilution) or anti-A-LAP antibody (1:1000 dilution). Membranes were washed with PBS and incubated with a secondary antibody. After washing with PBS, membranes were treated with an ECL blotting detection system (Amersham Pharmacia Biotech, New Jersey).
For subcellular fractionation, placental tissues were homogenized in a homogenization buffer $(0.25 \mathrm{M}$ sucrose; 20 mm Tris- $\mathrm{HCl}$, pH 7.5; 150 mm NaCl; 1 mm PMSF; and $10 \mu \mathrm{M}$ pepstatin). The microsomal and cytosolic fractions were isolated as previously described (Nakanishi et al, 2000; Oya et al, 1976). Briefly, the tissue homogenate was fractionated by differential centrifugation at $600 \times g$ for 10 minutes, $8000 \times g$ for 10 minutes, and finally $105,000 \times g$ for 60 minutes. Then, the resultant supernatant was used as a cytosol fraction, and the resultant pellet was resuspended in homogenizing buffer containing 1\% NP-40 and used as a microsome fraction. Immunoblot analysis for $\mathrm{P}$-LAP in each fraction was performed using the same procedure.

\section{Immunohistochemistry}

Immunohistochemical staining was performed using the avidin-biotin immunoperoxidase technique as described previously (Ino et al, 2000). Sections were cut at a thickness of $4 \mu \mathrm{m}$. For heat-induced epitope retrieval, deparaffinized sections in $0.01 \mathrm{M}$ citrate buffer were treated three times for 5 minutes at $95^{\circ} \mathrm{C}$ in a microwave oven. Endogenous peroxidase activity was blocked by incubation with $3 \% \mathrm{H}_{2} \mathrm{O}_{2}$ in methanol for 20 minutes. After nonspecific $\mathrm{lg}$ binding was blocked by incubation with $10 \%$ normal goat serum, primary antibodies to CK7 (1:200 dilution), A-LAP (1:200), and P-LAP (1:200) were applied for 1 hour at room temperature. After washing with PBS, secondary biotinylated antibody was applied for 30 minutes. After washing with PBS, the streptavidin-peroxidase conjugate was applied for 15 minutes. Finally, sections were incubated with 3-amino-9-ethylcarbazole (AEC, Nichirei, Tokyo, Japan) for 10 minutes and were counterstained with Mayer's hematoxylin. Normal rabbit IgG was used as a negative control. Immunostaining intensity was scored semi-quantitatively based on the percent positivity of stained cells on a four-tiered scale as follows: -, negative (no positive cells); +/-, weakly or focally positive ( $<30 \%$ positive cells); + , moderately positive (30\% to $70 \%$ positive cells); and ++ , strongly or diffusely positive ( $>70 \%$ positive cells). In each case, at least three different areas were evaluated and the mean of the results was considered the expression intensity score. Scoring was performed twice, independently by two investigators.

\section{Immunofluorescence Staining}

Cells were cultured in four-well chamber glass slides (LabTek, Nunc Inc., Naperville, Illinois) in culture medium. The cells were fixed with $4 \%$ paraformaldehyde for 30 minutes at $4^{\circ} \mathrm{C}$, washed with PBS, and permeabilized in PBS containing $0.3 \%$ Triton X-100 for 5 minutes. Slides were incubated in blocking solution containing $2 \%$ BSA for 1 hour, and incubated with anti-P-LAP polyclonal antibody or anti-KDEL (ERretention signal sequence) mAb (Stressgen Biotechnologies, Victoria, Canada) for 1 hour at room temperature. After washing with PBS, the slides were 
incubated with secondary antibodies, Rhodamineconjugated goat antirabbit IgG (Santa Cruz Biotechnology, Santa Cruz, California) or FITC-conjugated goat antimouse IgG (Santa Cruz Biotechnology). After washing with PBS, fluorescence was visualized by confocal laser microscopy.

\section{References}

Akada T, Yamazaki T, Miyashita H, Niizeki O, Abe M, Sato A, Satomi S, and Sato Y (2002). Puromycin insensitive leucylspecific aminopeptidase (PILSAP) is involved in the activation of endothelial integrins. J Cell Physiol 193:253-262.

Blaschitz A, Weiss U, Dohr G, and Desoye G (2000). Antibody reaction patterns in first trimester placenta: Implications for trophoblast isolation and purity screening. Placenta 21:733741.

Caniggia I, Mostachfi H, Winter J, Gassmann M, Lye SJ, Kuliszewski M, and Post M (2000a). Hypoxia-inducible factor-1 mediates the biological effects of oxygen on human trophoblast differentiation through TGFbeta3. J Clin Invest 105:577-587.

Caniggia I, Winter J, Lye SJ, and Post M (2000b). Oxygen and placental development during the first trimester: Implications for the pathophysiology of pre-eclampsia. Placenta 21(Suppl A):25-30.

Cassoni P, Sapino A, Munaron L, Deaglio S, Chini B, Graziani A, Ahmed A, Bussolati G (2001). Activation of functional oxytocin receptors stimulates cell proliferation in human trophoblast and choriocarcinoma cell lines. Endocrinology 142:1130-1136.

Chakraborty C, Barbin YP, Chakrabarti S, Chidiac P, Dixon SJ, Lala PK (2003). Endothelin-1 promotes migration and induces elevation of $\left[\mathrm{Ca}^{2+}\right]$ and phosphorylation of MAP kinase of a human extravillous trophoblast cell line. Mol Cell Endocrinol 201:63-73.

Chou CS, Zhu H, Shalev E, MacCalman CD, Leung PCK (2002). The effects of gonadotropin-releasing hormone $(\mathrm{GnRH})$ I and $\mathrm{GnRH}$ II on the urokinase-type plasminogen activator/plasminogen activator inhibitor system in human extravillous cytotrophoblasts in vitro. J Clin Endocrinol Metab 87:5594-5603.

Cooper AC, Robinson G, Vinson GP, Cheung WT, and Pipkin FB (1999). The localization and expression of the reninangiotensin system in the human placenta throughout pregnancy. Placenta 20:467-474.

Damsky CH, Fitzgerald ML, and Fisher SJ (1992). Distribution patterns of extracellular matrix components and adhesion receptors are intricately modulated during first trimester cytotrophoblast differentiation along the invasive pathway, in vivo. J Clin Invest 89:210-222.

Damsky CH, Librach C, Lim KH, Fitzgerald ML, McMaster MT, Janatpour M, Zhou Y, Logan SK, and Fisher SJ (1994). Integrin switching regulates normal trophoblast invasion. Development 120:3657-3666.

Floridon C, Nielsen O, Holund B, Sunde L, Westergaard JG, Thomsen SG, and Teisner B (2000). Localization of E-cadherin in villous, extravillous and vascular trophoblasts during intrauterine, ectopic and molar pregnancy. Mol Hum Reprod 6:943-950.

Hattori A, Kitatani K, Matsumoto H, Miyazawa S, Rogi T, Tsuruoka N, Mizutani S, Natori Y, and Tsujimoto M (2000).
Characterization of recombinant human adipocyte-derived leucine aminopeptidase expressed in Chinese hamster ovary cells. J Biochem 128:755-762.

Hattori A, Matsumoto H, Mizutani S, and Tsujimoto M (1999). Molecular cloning of adipocyte-derived leucine aminopeptidase highly related to placental leucine aminopeptidase/ oxytocinase. J Biochem 125:931-938.

Huppertz B, Kertschanska S, Demir AY, Frank H-G, and Kaufmann P (1998). Immunohistochemistry of matrix metalloproteinases (MMP), their substrates, and their inhibitors (TIMP) during trophoblast invasion in the human placenta. Cell Tissue Res 291:133-148.

Ino K, Suzuki T, Uehara C, Nagasaka T, Okamoto T, Kikkawa F, and Mizutani S (2000). The expression and localization of neutral endopeptidase $24.11 /$ CD10 in human gestational trophoblastic diseases. Lab Invest 80:1729-1738.

Kajiyama H, Kikkawa F, Khin EE, Shibata K, Ino K, and Mizutani S (2003). Dipeptidyl peptidase IV overexpression induces up-regulation of E-cadherin and tissue inhibitors of matrix metalloproteinases, resulting in decreased invasive potential in ovarian carcinoma cells. Cancer Res 63:22782283.

Kajiyama H, Kikkawa F, Suzuki T, Shibata K, Ino K, and Mizutani S (2002). Prolonged survival and decreased invasive activity attributable to dipeptidyl peptidase IV overexpression in ovarian carcinoma. Cancer Res 62:2753-2757.

Kaufmann P and Castellucci M (1997). Extravillous trophoblast in the human placenta: A review. Trophoblast Res 10:21-65.

Keller SR, Scott HM, Mastick CC, Aebersold R, and Lienhard GE (1995). Cloning and characterization of a novel insulinregulated membrane aminopeptidase from Glut4 vesicles. J Biol Chem 270:23612-23618.

Li X, Shams M, Zhu J, Khalig A, Wilkes M, Whittle M, Barnes $N$, and Ahmed A (1998). Cellular localization of $A T_{1}$ receptor mRNA and protein in normal placenta and its reduced expression in intrauterine growth restriction. Angiotensin II stimulates the release of vasorelaxants. J Clin Invest 101: 442-454.

Miyashita H, Yamazaki T, Akada T, Niizeki O, Ogawa M, Nishikawa S, and Sato $Y$ (2002). A mouse orthologue of puromycin-insensitive leucyl-specific aminopeptidase is expressed in endothelial cells and plays an important role in angiogenesis. Blood 99:3241-3249.

Mizutani S, Goto K, Itakura A, Furuhashi M, Kurauchi O, Kikkawa F, and Tomoda Y (1994). Physiological role of placental proteases: Interaction between pregnancy-induced bioactive peptides and proteases. Endocr J 41(Suppl):93104.

Nagasaka T, Nomura S, Okamura M, Tsujimoto M, Nakazato H, Oiso Y, Nakashima N, and Mizutani S (1997). Immunohistochemical localization of placental leucine aminopeptidase/ oxytocinase in normal human placental, fetal and adult tissues. Reprod Fertil Dev 9:747-753.

Nakamura $\mathrm{H}$, Itakura A, Okamura M, Ito M, Iwase A, Nakanishi Y, Okada M, Nagasaka T, and Mizutani S (2000). Oxytocin stimulates the translocation of oxytocinase of human vascular endothelial cells via activation of oxytocin receptors. Endocrinology 141:4481-4485. 
Nakanishi $\mathrm{Y}$, Nomura S, Okada M, Ito T, Katsumata $\mathrm{Y}$, Kikkawa F, Hattori A, Tsujimoto M, and Mizutani S (2000). Immunoaffinity purification and characterization of native placental leucine aminopeptidase/oxytocinase from human placenta. Placenta 21:628-634.

Nomura M, Tsukahara S, Ando H, Katsumata Y, Okada M, Itakura A, Nomura S, Kikkawa F, Nagasaka T, and Mizutani S (2002). Differential distribution of placental leucine aminopeptidase/oxytocinase and aminopeptidase $A$ in human trophoblasts of normal placenta and complete hydatidiform mole. Placenta 23:631-639.

Oya M, Wakabayashi T, Yoshino M, and Mizutani S (1976). Subcellular distribution and electrophoretic behavior of aminopeptidase in human placenta. Physiol Chem Physics 8:327-335.

Page NM, Kemp CF, Butlin DJ, and Lowry PJ (2002). Placental peptides as markers of gestational disease. Reprod 123: 487-495.

Pijnenborg R, Bland JM, Robertson WB, and Brosens I (1983). Uteroplacental arterial changes related to interstitial trophoblast migration in early human pregnancy. Placenta 4:397-414.

Rogi T, Tsujimoto M, Nakazato H, Mizutani S, and Tomoda $Y$ (1996). Human placental leucine aminopeptidase/ oxytocinase: A new member of type II membrane-spanning zinc metallopeptidase family. J Biol Chem 271:56-61.

Ross SA, Scott HM, Morris NJ, Leung WY, Mao F, Lienhard GE, and Keller SR (1996). Characterization of the insulinregulated membrane aminopeptidase in 3T3-L1 adipocytes. J Biol Chem 271:3328-3332.

Saric T, Chang SC, Hattori A, York IA, Markant S, Rock KL, Tsujimoto M, and Goldberg AL (2002). An IFN-gammainduced aminopeptidase in the ER, ERAP1, trims precursors to MHC class I-presented peptides. Nat Immunol 3:11691176.

Sato Y, Fujiwara H, Higuchi T, Yoshioka S, Tatsumi K, Maeda M, and Fujii S (2002). Involvement of dipeptidyl peptidase IV in extravillous trophoblast invasion and differentiation. J Clin Endocrinol Metab 87:4287-4296.

Serwold T, Gonzalez F, Kim J, Jacob R, and Shastri N (2002). ERAAP customizes peptides for MHC class I molecules in the endoplasmic reticulum. Nature 419:480-483.
Shih leM, Hsu MY, Oldt RJ 3rd, Herlyn M, Gearhart JD, and Kurman RJ (2002). The role of E-cadherin in the motility and invasion of implantation site intermediate trophoblast. Placenta 23:706-715.

Shih IM and Kurman RJ (1996). Expression of melanoma cell adhesion molecule in intermediate trophoblast. Lab Invest 75:377-388.

Suzuki Y, Shibata K, Kikkawa F, Kajiyama H, Ino K, Nomura S, Tsujimoto M, and Mizutani S (2003). Possible role of placental leucine aminopeptidase in the antiproliferative effect of oxytocin in human endometrial adenocarcinoma. Clin Cancer Res 9:1528-1534.

Tsujimoto M, Mizutani S, Adachi H, Kimura M, Nakazato $\mathrm{H}$, and Tomoda $Y$ (1992). Identification of human placental leucine aminopeptidase as oxytocinase. Arch Biochem Biophys 292:388-392.

Uehara C, Ino K, Suzuki T, Kajiyama H, Kikkawa F, Nagasaka $\mathrm{T}$, and Mizutani S (2001). Upregulation of neutral endopeptidase expression and enzymatic activity during the differentiation of human choriocarcinoma cells. Placenta 22:540549.

Vinson GP, Saridogan E, Puddefoot JR, and Djahanbakhch O (1997). Tissue renin-angiotensin system and reproduction. Human Reprod 12:651-662.

Xia Y, Wen HY, and Kellems RE (2002). Angiotensin II inhibits human trophoblast invasion through AT1 receptor activation. J Biol Chem 277:24601-24608.

Zhou Y, Fisher SJ, Janatpour M, Genbacev O, Dejana E, Wheelock M, and Damsky CH (1997). Human cytotrophoblasts adopt a vascular phenotype as they differentiate. A strategy for successful endovascular invasion? J Clin Invest 99:2139-2151.

Zhou Y, Genbacev O, Damsky CH, and Fisher SJ (1998). Oxygen regulates human cytotrophoblast differentiation and invasion: Implications for endovascular invasion in normal pregnancy and in pre-eclampsia. J Reprod Immunol 39:197213. 